\title{
Technique possibilities for volumetric assessment of intracranial aneurysms
}

\author{
A. Chiriac, B. Iliescu, N. Dobrin, I. Poeată \\ Clinic of Neurosurgery, "Gr.T. Popa" University of Medicine and Pharmacy Iasi \\ "Prof. Dr. N. Oblu" Clinical Emergency Hospital, Iaşi, Romania
}

\begin{abstract}
Volume measurement techniques of intracranial aneurysms from 3D rotational angiography vary on different factor settings and, therefore, are operator-dependent. The purpose of this study was to evaluate the application and the precision of ellipsoidal approximation (mathematics and computer technique) and software methods to measure intracranial aneurysms volume starting from planar (DR and SD) and 3dimensional (3D) angiographic images. The reliability of the methods was statistically compared in a clinical setting of 42 angiograms and 100 measurements performed by the same users. This study suggests that both techniques could be used for clinical applications with similar efficiency results.
\end{abstract}

The 3D rotational angiography is an increasingly used method for cerebral aneurysms treatment planning. This complex imagistic method permits a better evaluation of cerebral aneurysms anatomy by an improved delimitation of vessels and aneurysms contours. Moreover, the 3D dataset can be used to calculate aneurysm volume. It is known the important role of aneurysm volume calculation in coil embolization treatment. To assess an optimal degree of coils aneurysm packing, it has become imperative to determine preoperatively the volume of the aneurysm to be embolized.

\section{Methods}

Retrospective assessment of aneurysm volume was achieved with two measurement methods by using twodimensional copies or 3D reconstruction images (digital or printed form in sagittal and coronal angiography section) obtained with a Siemens Artis Angiograph with rotational digital subtraction possibilities for 42 intracranial aneurysms.

\section{Ellipsoid approximation method}

This method of volume measuring is based on the assumption that the aneurysms shape could be geometrical approximated with an ellipse. An ideal ellipsoid volume can be calculated using the mathematical relationship between the length (L) large diameter (D) and small diameter (d).

For these measurement techniques we have gone on the premise that exposure of an aneurysm with a two-dimensional angiography is performed with the vascular lesion located exactly or very close to isocenter of mobile $\mathrm{C}$-arm. The distance between X-ray source and image amplifier was maximum $(117 \mathrm{~cm}$ and $130 \mathrm{~cm}$ for exposures AP [anterior-posterior] that 
exposures profile) to ensure that there is plenty of room to manoeuvre both the patient and the mobile C-arm during the procedure. Diameter of the image intensifier entrance was known for all angiography.

Two-dimensional images obtained by contrast injection and fluoroscopic exposures were printed on transparent film or paper. Also the digital images in DICOM format obtained through angiography $\mathrm{DR}$ and $\mathrm{SD}$ regime were processed using e-Film Medical software in multiplanar reconstruction (MPR).

Mathematical technique - By this technique the printed image of aneurysm was measured with a ruler directly on film, while digital images were measured using e-Film Medical software that allow a direct measurement of aneurysm size (Figure 1).

Aneurysm volume was estimated by using mathematical equation corresponding to the formula of volume of a perfect ellipse:

$$
\mathrm{V}_{\mathrm{c} a 1}=\frac{4}{3} \pi \frac{L}{2} \frac{D}{2} \frac{\mathrm{d}}{2}=\pi \frac{L D \mathrm{~d}}{6}
$$


Figure 1 Aneurysm measuring on E-film multiplanar reconstruction

Computerized technique - By the second technique the surface reconstruction of a volume can be achieved by establishing correspondence between points on two images. The relationship between the two images defined by the connection points is used to reproduce the epipolar geometry and create the epipolar images used for volume generation. Materialization of connection points can be automatically or interactively achieved. Automatic generation of connection points - ENVI algorithm can automatically generate the contact points, based on figure characteristics from the image. Choosing the option of interactive definition of connection points requires a manually definition of them between the two stereo images. Automatic generation control contains specific parameters that correctly defined can lead to a right-left correlation of the two images as real as possible and finally to extract a volume close to reality.

Epipolar geometry constrains the epipolar points that lie on an epipolar line in the left image to be projected on the epipolar line corresponding in the image on the right. Fundamental matrix is collinearity that offer a correlation between the epipolar lines and allows us to establish the epipolar line equation of the right image corresponding to a given point in left image and vice versa. Fundamental matrix is calculated from one or more pairs of images with the same characteristics of magnification and focusing.

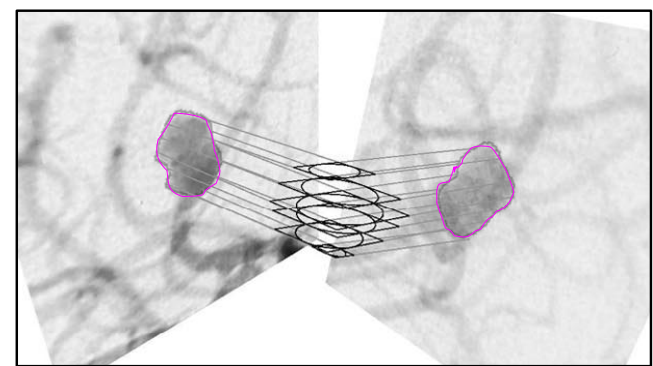

Figure 2 Aneurysm measuring by epipolar geometry 
DOI: 10.2478/v10282-012-0006-1

Thus, the aneurysm image on film or print paper is scanned using an Epson Perfection V700 Photo scanner at around 1000 x 1000 pixels. These digitized images were calibrated for the magnification and angle of entry of X-ray flux and then exposed to the same three-dimensional coordinate system. Adjust brightness / contrast and demarcation contours were used for manual segmentation of the aneurysm. After segmentation of the aneurysm in two projections, a computer program (AutoCAD) was used to reconstruct three-dimensional aneurysm as a superposition of several ellipses. Each ellipse is reconstructed based on four points corresponding to intersection of the epipolar plan with the outline aneurysm in both projections. Numerical integration of the multiple overlapping ellipses provides the estimated volume of aneurysm (Figure $2)$. Aneurysm is reconstructed by the superposition of at least 60 ellipses.

\section{D-generation Softwear methods}

The images acquisition for 3D reconstruction of vessels is done by using an 3D InSpace acquisition program option. This is a basic technique for $3 \mathrm{D}$ reconstructing data set form acquired $2 \mathrm{D}$ imaging in Dynavision rotational angiography mode. By this protocol images acquisition a series of 150 to 200 axial images are acquired in a period of 5-8 seconds.

Dynavision is a system-integrated solution for 3D imaging reconstruction in rotational angiography. Dynavision acquisition for the softwaer InSpace 3D benefits by predefined setup parameters. 3D DSA radiological parameters are preset as: the tube voltage $90 \mathrm{kV}$, tube current in $173 \mathrm{~m}$, pulsed $3.9 \mathrm{~ms}$, focusing 0 , frame rate $10 \mathrm{p} / \mathrm{s}$. Dynavision generates exposure under extremely precise radiation emission angles, providing optimal overlap of mask and contrast images, even at their low frame rates and high speed rotation. Also ensure minimize radiation dose to the patient, reduced examination time and improve image quality and patient comfort.

The imaging projections obtained on the basic computer system are transferred automatically to the post-processing computer unit (syngo Workplace-syngo Fastview) by selection of SEND to 3D command. Thus, the 3D images are automatically reconstructed and displayedon the monitor unit syngo Workplace. For the aneurysm volume measurement the Volume Analysis command is selected (Figure 3). A new window it will open requesting a manual contrast / brightness adjustment that will allow a clear highlight of the vascular tree.

Then, there will be a manually demarcation of the volume of interest to be measured (aneurysmal neck and sac) by selecting Inspace YOU command. Finally, to get automatic calculation of anuerysm volume the Compute Volume command will be selected. The volume of demarcated vascular area is quantified by integration of all pixels enrolled in 2D vascular contour. The result is visualised in four windows on the Workplace syngo monitor (Figure 3).

\section{Imaging technique validation}

For the beginning, we had to establish if the angiographic image acquisition system has a similar precision for 2D images (face, profile) and 3D images and if it's sufficiently precise to allow accurate volumetric measurement. Thus, our angiographic system (Siemens AXIOM Artis DFA, Germany) was tested by using four steel balls with different diameters (shall appear 1). 

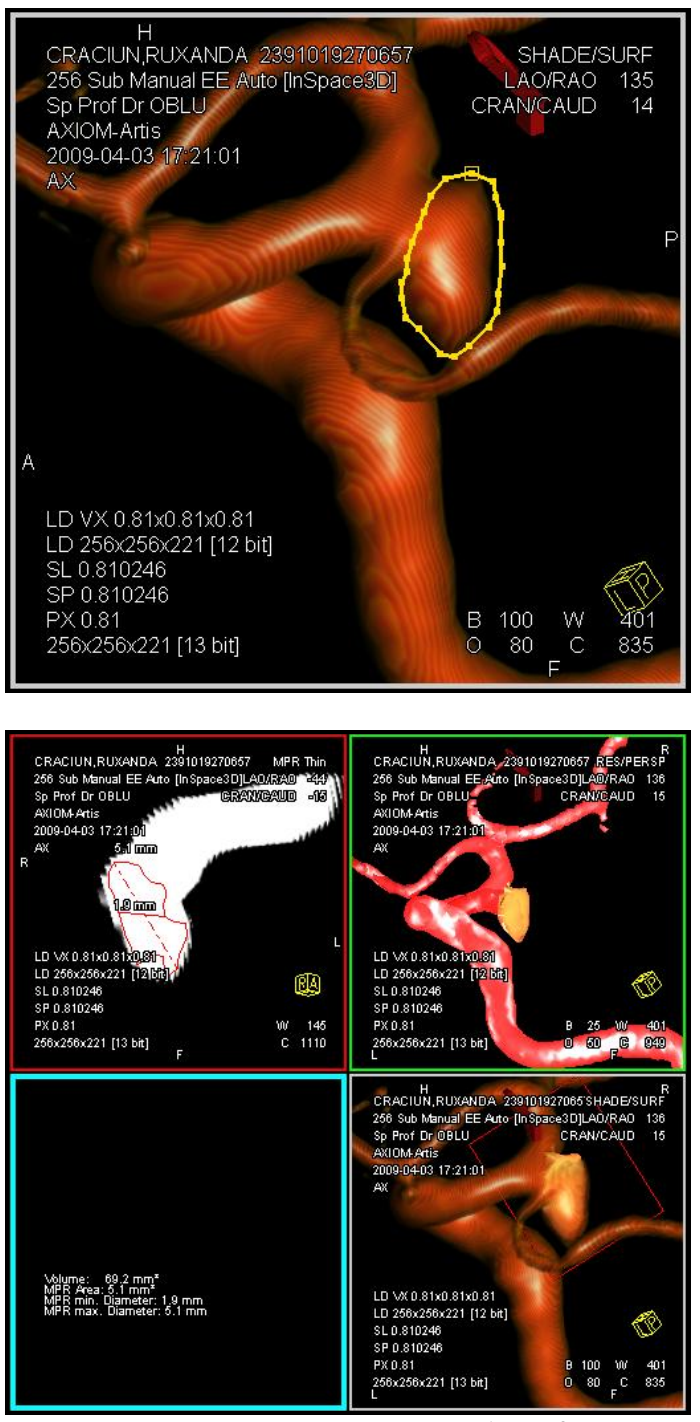

Figure 3 Aneurysm measuring by software methods

Their diameters were chosen in order to cover most of the dimensional limits of intracranial aneurysms.

Thus, in a first phase metal balls were accurately measured with a micrometer with an accuracy of $0.01 \mathrm{~mm}$ (Figure $4 \mathrm{~A}, \mathrm{~B}$, $\mathrm{C}, \mathrm{D})$. The values obtained were considered the actual dimensional values.

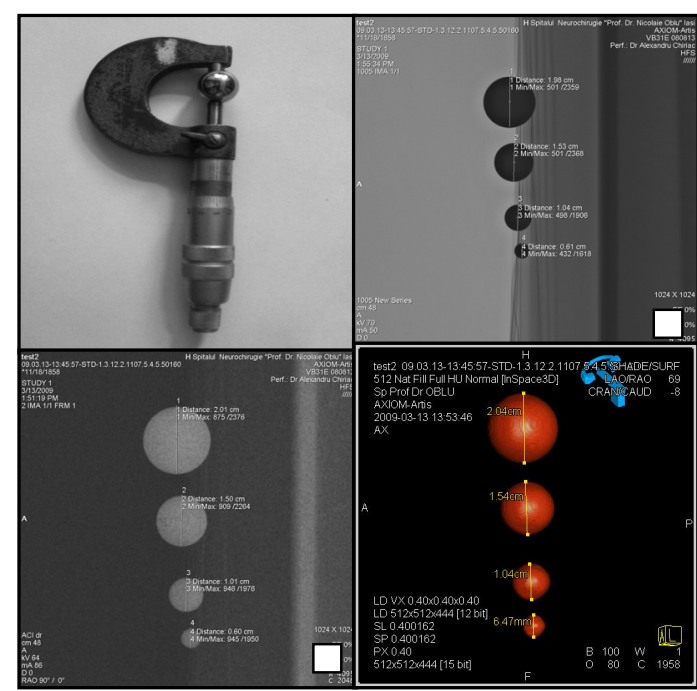

Figure 4 Aneurysm measuring by micrometer, and on DR, SD and 3D images

TABEL 1

\begin{tabular}{|c|c|c|c|c|}
\hline \multirow[t]{2}{*}{$\begin{array}{l}\mathrm{Nr} . \\
\text { test }\end{array}$} & \multirow[t]{2}{*}{$\begin{array}{c}\text { Diametru } \\
\text { prin masurare } \\
\text { cu } \\
\text { micrometru } \\
(\varnothing \mathrm{mm})\end{array}$} & \multicolumn{2}{|c|}{$\begin{array}{l}\text { Diametru prin } \\
\text { masurare pe } \\
\text { achiziţie } \\
\text { imagistica plană } \\
\text { (Ømm) }\end{array}$} & \multirow[t]{2}{*}{$\begin{array}{c}\text { Diametru } \\
\text { prin masurare } \\
\text { pe achiziţie } \\
\text { imagistica } \\
\text { 3D }(\varnothing \mathrm{mm})\end{array}$} \\
\hline & & $\mathrm{DR}$ & SD & \\
\hline \begin{tabular}{|l|} 
Bila 1 \\
\end{tabular} & 6,45 & 6,1 & 6,0 & 6.27 \\
\hline Bila 2 & 10,25 & 10,4 & 10,1 & 10 , \\
\hline \begin{tabular}{|l} 
Bila 3 \\
\end{tabular} & 15,05 & 15,3 & 15.0 & \\
\hline Bila 4 & 20,45 & 19,8 & 20.1 & 20,4 \\
\hline
\end{tabular}

After that, balls were investigated by planar angiographic imaging acquisition 2D (face and profile) and 3D, using the same acquisition parameters as for routine clinical investigations.

2D angiographic imaging acquisition was made in DR (direct) and SD (digital subtraction) modes (Figure 3). Four ball diameter values obtained by direct measurement in 2D (DR and SD) and 3D are presented in Table 1.

Discrepancy (measurement error) between the real volume and that obtained by angiographic imaging acquisition varied upward or downward from $0.2 \%$ for ball 
DOI: $10.2478 / \mathrm{v} 10282-012-0006-1$

test of $20.45 \mathrm{~mm}$ to $2.9 \%$ for ball test of $6.45 \mathrm{~mm}$. These small discrepancies obtained were considered statistically nonsignificant. The method of percutaneous angiographic acquisition and image processing unit allows us to obtain angiographic images with near-realdimensional features.

\section{Statistic analysis}

In this study we statistically compared each of the two methods of analysis in each measurement techniques by nonparametric tests because the scores of subjects with measurements is not a normal distribution (Gaussian). Statistical test applied to verify differences in overall was non-parametric Friedman test for more than two sample pairs (with repeated measurements, because each subject was measured by all three analysis techniques: ellipsoidal approximation mathematical / computer technique and software) and when there were found global significant differeces between the three

measurments techniques, nonparametric Wilcoxon test of was applied for two paired samples (two repeated measurements) to compare two by two analysis techniques applied in each measurmet methods used.

\section{Results}

Mean of patient's age from the study group was 51.19 years (aged between 22 and 69 years). Gender distribution showed a dominance of females at a ratio of 2:1. Distribution depending on location of aneurysms show a dominance of lesions from the anterior circulation (13 AntCoA, 10 MCA, 4 ICAbif, 3 Ophthalmic, PericalosalA 2) to those of the posterior circulation (7 PostCoA, 2 Basilar tip, one pica). Average values of measured aneurismal volume were $398.1 \mathrm{~mm} 3$ for ellipsoidal approximation method by mathematical technique, $320.4 \mathrm{~mm} 3$ for ellipsoidal approximation method by computer technique of and $320.1 \mathrm{~mm} 3$ for software measurement method. Statistical correlation between means aneurysm volumes depending on the method of measurement related to their location showed that the best correlation was for the location of the Basilar tip A and the weakest for the location of ophthalmic A (Table 2, Chart).

Based on statistical analysis obtained from the Friedman test we found statistically significant differences at $\mathrm{p} £$ 0,05 threshold between the three techniques of analysis $\left[\chi^{2}(2)=8.714, \mathrm{p}=\right.$ 0.013] (Annex 6).

Test Statistics ${ }^{\text {a }}$

\begin{tabular}{|l|r|}
\hline N & 42 \\
Chi-Square & 8.714 \\
df & 2 \\
Asymp. Sig. & .013 \\
\hline
\end{tabular}

a. Friedman Test

TABEL 2

\begin{tabular}{|c|c|c|c|}
\hline localization & $\begin{array}{c}\text { Ellipsoidal } \\
\text { approximation } \\
- \text { mathematical } \\
\text { technique }\end{array}$ & $\begin{array}{c}\text { Ellipsoidal } \\
\text { approximation } \\
- \text { computer } \\
\text { technique }\end{array}$ & $\begin{array}{c}\text { Software } \\
\text { methods }\end{array}$ \\
\hline ACM & 341,7726 & 314,7585 & 314,4954 \\
\hline ACoAnt & 116,0076 & 127,9095 & 128,0657 \\
\hline A.CoPost & 724,6029 & 533,1759 & 532,3124 \\
\hline Bif.ACI & 199,7813 & 186,2480 & 185,7411 \\
\hline A.Oft & 1407,4156 & 1008,4773 & 1006,7750 \\
\hline A.Perical & 296,3897 & 237,2902 & 236,3053 \\
\hline A Vf.Bazil & 107,0950 & 108,1665 & 108,5917 \\
\hline PICA & 195,0467 & 152,3627 & 150,7513 \\
\hline
\end{tabular}


To check significant differences between the three techniques of analysis we applied the Wilcoxon test. The results were as follows: - there were significant differences at $\mathrm{p} £ 0,05$ threshold between ellipsoid approximation method by mathematical technique and software method $[z=2.107$, $\mathrm{p}=0.035]$, meaning that measurements by ellipsoid approximation by mathematical technique are significantly higher compared with measurements obtained by software; there are significant differences at $\mathrm{p} £ 0,05$ threshold between ellipsoid approximation method by mathematical technique and ellipsoid approximation method by computer technique $[\mathrm{z}=2.169, \mathrm{p}=$ $0.030]$, meaning that measurements by ellipsoid approximation method by mathematical technique are significantly higher compared with measurements obtained ellipsoid approximation method by computer technique; - there were no statistically significant differences at $\mathrm{p} £$ 0,05 threshold between method software and ellipsoid approximation method by computer technique $[\mathrm{z}=1.532, \mathrm{p}=$ $0.126]$.

Rank

\begin{tabular}{|l|r|}
\hline & Mean Rank \\
\hline Ellipsoidal approximation & 2.33 \\
- mathematical technique & \\
Ellipsoidal approximation & 1.98 \\
- computer technique & \\
Software technique & 1.69 \\
\hline
\end{tabular}

Test Statistics ${ }^{b}$

\begin{tabular}{|l|r|r|r|}
\hline & $\begin{array}{c}\text { Ellipsoidal } \\
\text { approximation } \\
\text { - mathematical } \\
\text { technique }\end{array}$ & $\begin{array}{c}\text { Ellipsoidal } \\
\text { approximation } \\
\text { - computer } \\
\text { technique }\end{array}$ & $\begin{array}{l}\text { Software } \\
\text { technique }\end{array}$ \\
\hline Z & $-2.169^{\mathrm{a}}$ & $-2.107 \mathrm{a}$ & $-1.532^{\mathrm{a}}$ \\
Asymp. Sig. (2-tailed) & .030 & .030 & .126 \\
\hline
\end{tabular}

\section{Discussions}

These discrepancies have been highlighted statistics on the difference "imaging behaviour" between different types of aneurysms after injection of the contrast in interventional procedures. Also, precision of planar angiographic imaging acquisition and of the $3 \mathrm{D}$ is usually estimated by manual injections of contrast, known that speed of injection is a relative variable factor. In addition, it is important to note that the measurement volume purchases based on angiographic imaging may vary depending on how the display was chosen by the operator. For standardization of angiographic images of aneurysms, window settings should be adjusted for the purposes of background suppression artefacts and quality of aneurysm contour edges.

$3 \mathrm{D}$ reconstructions based on rotational angiographic images are influenced by technical and physiological limitations of the method of acquisition. Possible distortion of system acquisition due to equipment vibration, dilution of contrast medium due to blood flow dynamics and data degradation due to pulsation, amplified respiratory movements and heterogeneity of surrounding tissue can reduce image quality. These degradations generally cause unclear imaging data, resulting in increased difficulty in segmentation of structures of interest, such as aneurysmal contours. To maximize the aneurysmal sac filling with contrast agent throughout the rotational cycle, it has been shown that the quality of the contrast bolus injection (volume, flow rate) improves visualization of aneurysms on 3D reconstruction compared with continuous flow technique.

Visualisation of saccular aneurysms by $3 \mathrm{D}$ reconstruction of angiographic imaging acquisition dates is directly related to intraaneurysmal hemodynamic, which in turn is 
DOI: $10.2478 / \mathrm{v} 10282-012-0006-1$

closely related to the geometric relationship between the aneurysm and parent vessel. In case of the bifurcation or terminal aneurysms hemodynamic experiments described a rapidly intra-aneurysmal circulation without the formation of vortices and stasis, while for lateral aneurysms were described a regular flow model with inflow to the distal extremity of the neck, vortex flow in the centre and reflux along the aneurysm wall. While the bifurcation aneurysms (such as those described often in the middle cerebral artery, communicating artery pericallosal, carotid bifurcation and basilar tip) can be identified with a highly accurate imaging, lateral aneurysms viewing (internal carotid artery in cavernous segment, ophthalmic and other supraclinoid aneurysms) with small neck can sometimes be affected by incomplete filling with contrast.

\section{Conclusions}

Restrictions mentioned above may be sources of measurement errors of aneurysmal volume. However, discrepancies identified between the real volume and that obtained by angiographic imaging for small and medium size aneurysms were lower, the methods and techniques measurement are considered valid for clinical use.

\section{References}

1. Bland JM, Altman DG. Comparing methods of measurement: why plotting difference against standard method is misleading. Lancet 1995;346:1085-1087;

2. Javier Oliva'n Besco's, Marjan J. Slob, Cornelis H. Slump, Menno Sluzewski, and Willem Jan van Rooi (2005) Volume Measurement of Intracranial Aneurysms from 3D Rotational Angiography: Improvement of Accuracy by Gradient Edge Detection, Am J Neuroradiol 26:2569-2572;

3. Tamatani S, Ito $\mathrm{Y}$, Abe $\mathrm{H}$, Koike $\mathrm{T}$, Takeuchi $\mathrm{S}$, Tanaka R. Evaluation of the stability of aneurysms using detachable coils: correlation between stability of aneurysms and embolized volume of aneurysms. AJNR Am J Neuroradiol 2002; 23:762-767;

4. Vincent Costalat, Igor Lima Maldonado, Olivier Strauss, Alain Bonafé (2011) Toward accurate volumetry of brain aneurysms: Combination of an algorithm for automatic thresholding with a 3D eraser tool, J Neurosci Methods 7. 